\title{
Histopathological Study of Non-Cirrhotic Portal Fibrosis (NCPF) With Special Emphasis on Advanced Fibrosis
}

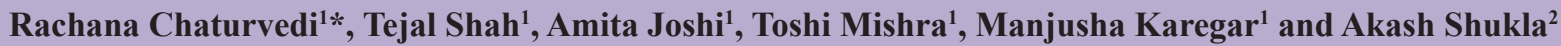 \\ ${ }^{1}$ Department of Pathology Seth G.S. Medical College \& KEM Hospital, Mumbai, India \\ ${ }^{2}$ Department of Gastroenterology, Seth G.S. Medical College \& KEM Hospital, Mumbai, India
}

\begin{abstract}
Background: Non-cirrhotic portal hypertension includes primarily the cases of non-cirrhotic portal fibrosis (NCPF) and extra-hepatic portal vein obstruction, both showing no significant parenchymal disease. NCPF is a disease of uncertain etiology characterized by periportal fibrosis with small and medium sized portal vein branches involvement. Most study done in the past are clinical, hence we decided to analyse its histo-pathological features with clinical correlation.

Methods: This is four and half year's retrospective study of liver biopsies of clinically diagnosed patients of NCPF. Fifteen patients of EHPVO served as controls. Biopsies were stained with Haematoxylin and eosin, reticulin and Masson's trichrome.

Result: Total 978 benign specimens (excluding space-occupying lesions) received, of which 45 diagnosed as NCPF (4.6\%). Most patients (68.88\%) were between 20-40 years (M:F ratio 1:1.5), commonest symptoms long-standing splenomegaly (95\%), anemia (78\%) and variceal bleeds (42\%) with duration varying from 3 months to 11 years. Commonest histopathological findings were portal tract fibrosis (77.78\%), phlebosclerosis $(68.89 \%)$, portal tract remnants $(24.44 \%)$, more frequently seen in NCPF than EHPVO, septal fibrosis was seen in five. Follow up available in $91.11 \%$ cases showed no mortality or liver failure, but significant morbidity due to repeated episodes of variceal bleeds and hypersplenism.

Conclusion: NCPF is still common in India. Distinctive features on histology are portal tract fibrosis, portal tract remnants, with phlebosclerosis which is being statistically significant when comparing with EHPVO. NCPF can show advanced fibrosis, can also present with features of decompensation mimicking cirrhosis, however its course remains stable with good long-term survival.
\end{abstract}

Keywords: PHT, NCPF, EHPVO, Phlebosclerosis, Portal Tract Fibrosis.

\section{Introduction}

Portal hypertension (PHT) can be cirrhotic and noncirrhotic. The diseases leading to non cirrhotic portal hypertension $(\mathrm{NCPH})$ are primarily vascular in nature and classified anatomically on the basis of site of resistance to blood flow, as pre-hepatic, hepatic and post-hepatic. Hepatic causes are further classified as pre-sinusoidal, sinusoidal and post-sinusoidal. Two main entities presenting with $\mathrm{NCPH}$ are non cirrhotic portal fibrosis (NCPF) and extra hepatic portal venous obstruction (EHPVO), both of which show no significant parenchymal dysfunction. ${ }^{[1,2,3]}$.

EHPVO is a childhood disorder, more commonly seen in developing countries. It is characterized by a chronic blockage of main portal vein leading to PHT and upper gastrointestinal bleeding. ${ }^{[4,5,6,7,8]}$ Histology can overlap with $\mathrm{NCPF}$, however, radiology shows portal cavernoma.

NCPF is a disorder of young adults and is clinically characterized by moderate to marked splenomegaly (disproportionate with average weight 723 gms.), with or without hypersplenism with patent hepatic and portal veins ${ }^{[2,3,9]}$ The portal pressure is comparable to that of other conditions causing PHT ${ }^{[10]}$ and the clinical features overlap with cirrhosis. The disease has been reported from all parts of worlds, more from the developing countries. ${ }^{[11,12,13,14,15]}$ where it is more common in young males ( $3^{\text {rd }}$ to $4^{\text {th }}$ decades) of low socioeconomic groups. ${ }^{[2,10,15,16,17]}$ Similar kind of disease known as idiopathic portal hypertension (IPH) in Japan and idiopathic non-cirrhotic portal hypertension (INCPH) in west, has female preponderance and presents later ( $4^{\text {th }}$ and $5^{\text {th }}$ decade). The etiology of NCPF is unknown and poorly understood. A number of hypotheses are postulated, and infections and prothrombotic states as etiology are commonly incriminated in the eastern and western patients, respectively. On gross examination, the portal vein is generally dilated with thickened walls. Autopsy series commonly show thrombosis in the medium and small (diameter $<300 \mathrm{um}$ ) portal vein branches. ${ }^{[3,8,11]}$

Incidence of NCPF is reduced these days, but it still remains one of the commoner causes of NCPH in India. Though many clinical studies have been done, but there are only few primarily describing the detailed histological features of NCPF. 
Ours is one of the largest public hospitals and 1800 bedded tertiary care centre in western India, which caters to the poor patients virtually free of cost and has a dedicated hepatology department. Hence, we decided to do the histopathological analysis of NCPF on liver biopsies with its clinical correlation and to know its burden in our setup.

\section{Materials and Methods}

This is a retrospective study of liver biopsies, received over the period of 4.5 years (January 2010 to June 2014), of clinical cases of NCPF, either diagnosed at our institute or referred from outside for further management.

Inclusion criteria: Clinical evidence of PHT (ii) Radiological examination (abdominal CT or Doppler Ultrasound) demonstrating patent portal and hepatic veins at initial diagnosis (iii) Negative markers including viral, autoimmune etc with normal copper studies (iv) No clinical/ histological evidence of known causes of non-cirrhotic PHT (other than NCPF) including hepatic schistosomiasis, congenital hepatic fibrosis, nodular regenerative hyperplasia etc.

Exclusion criteria: Space occupying/ infiltrative lesions.

Control: EHPVO patients, where clinical features are either non-classical or where biopsy done as a routine, at the time of splenectomy to rule out cirhhosis.

Relevant clinical and laboratory data were recorded from patient's files. All liver biopsies were fixed with $10 \%$ neutral buffered formalin. Serial step sections of 3 um thicknesses were prepared and stained with haematoxyllin and eosin (H\&E), reticulin, masson's trichrome, Periodic Schiff with diastase, orcein and Prussian blue stains. Biopsies were then evaluated for the following histological features-

i) Phlebosclerosis, defined as a portal vein with a reduced or completely sclerosed lumen, and was graded according to percentage of portal tracts affected $(0 \%$, $<50 \%$ and $>50 \%$ )

ii) Paraportal shunting vessels, defined as enlarged thin walled vessels outside but in close contact with PT.

iii) Aberrant vessels defined as, increased number of draining vessels inside the portal tract or lobular parenchymal (angiomatoid lesion).

iv) Portal tract remnants, defined as PT having size smaller than twice the diameter of bile duct, which is smaller than the size of the periportal surrounding hepatocytes.

v) Presence of dilated portal vein branch (normal being at least three times greater than the diameter of the bile duct, or the hepatic artery). v) Thin incomplete septae, defined as those ending blindly over the lobules

Staging of fibrosis was done as per Ishak's modification of hepatitis activity index (HAI) system for chronic hepatitis. ${ }^{[18,19,20]}$ Stage 1 and 2 were defined as lower stage and stage $\geq 3$ as higher stage fibrosis. Follow ups were done wherever available.

Continuous variables were compared using ANOVA, discrete variables by Fisher's exact test, and non-Gaussian variables by a non-parametric test. P-values $\leq 0.05$ was considered statistically significant when two groups are compared. $\alpha$ error was taken as 0.2 ( $80 \%$ accuracy).

Permission: from Institutional ethics committee.

\section{Result}

Of the total 978 cases of non-neoplastic livers received for histopathology (excluding benign space occupying lesions), 45 were of NCPF (28 transjugular, 16 percutaneous and 1 wedge biopsy).

Patients' age ranged from 18 to 60 years (mean age $34.3 \mathrm{yrs)}$ and most patients (75.5\%) presented between 20 to 40 years. There was slight female preponderance with male: female ratio being 1:1.5 (18 M, 27 F). The duration of symptoms varied from 3 months, to 11 years (patient referred from outside) and most common presenting symptoms (table 1) were long standing splenomegaly, esophageal varices with bleeding and anemia. Symptoms of decompensation such as ascites $(n=7,15.56 \%)$ and jaundice $(n=3,6.67 \%)$ were present in few. None had hepatic encephalopathy.

Amongst liver function tests, the aspartate aminotransferase (AST) levels were raised in $16(42.10 \%$, range 40 to $90 \mathrm{U} / \mathrm{L}$ ) while alanine amino-transferase (ALT) levels were raised in $6(15.79 \%$, range 42 to $69 \mathrm{U} / \mathrm{L})$. The direct bilirubin was raised in $9(20 \%)$ cases, hypoalbuminemia in $16(33.33 \%)$ and raised PT-INR in 14 patients $(31.1 \%$, range 1.1 to 1.8$)$. The endoscopy was available in $35(77.8 \%)$ and all these showed esophageal varices, with gastric varices in $2(4.44 \%)$ and features of portal gastropathy in $1(2.22 \%)$.

Among the histological findings (Table 1), the specific features like portal tract fibrosis, phlebosclerosis were more common (Figure 1) with other features (Figure 2) like portal tract remnants, portal tract approximation and short/ bridging septae (Figure 1). Nonspecific features of portal hypertension such as sinusoidal and portal vein dilatation, paraportal shunt vessels and increased vascular channels in portal tract and lobular parenchyma were seen in few and normal liver histology was found in $6(13.3 \%)$ cases. Of the cases with septal fibrosis ( $\mathrm{n}=5$, table 2$)$, one 
showed short septae (stage 1), three showed bridging septae (stage 3) and one showed bridging septae with occasional nodule formation (stage 5). We also attempted a clinical correlation of these cases with septal fibrosis. All patients were female with age ranged from 22 to $46 \mathrm{yrs}$ and duration of symptoms varied from 3 months to 4 years. The aminotransferase levels were minimally raised in 2 cases. They all presented with splenomegaly and anemia and two had esophageal varices. None had jaundice or ascites.

Follow up was available in 41(91.1\%) patients with duration varying from 6 months to 11 years. All patients were alive with near normal liver function tests, including

Table 1: Comparison of features of NCPF and EHPVO.

\begin{tabular}{|c|c|c|c|}
\hline Clinical and histological features & NCPF: $n(\%)$ & EHPVO: $n(\%)$ & p-value \\
\hline \multicolumn{4}{|l|}{ Clinical features } \\
\hline Splenomegaly & $43(95.56)$ & $15(100)$ & 1.00 \\
\hline Esophageal varices & $36(80)$ & $13(86.67)$ & 0.71 \\
\hline Variceal bleeding & $18(40)$ & $10(60.67)$ & 0.13 \\
\hline \multicolumn{4}{|c|}{ Histological features Specific Features } \\
\hline $\begin{array}{l}\text { PT fibrosis } \\
\text { Phlebosclerosis }\end{array}$ & $\begin{array}{l}35(77.78) \\
31(68.89)\end{array}$ & $\begin{array}{c}5(33.33) \\
1(6.62)\end{array}$ & $\begin{array}{c}0.0034 \\
<0.0001\end{array}$ \\
\hline $\begin{array}{l}\text { PT remnants } \\
\text { PT approximation } \\
\text { Septal fibrosis }\end{array}$ & $\begin{array}{l}11(24.44) \\
8(17.78) \\
5(11.11)\end{array}$ & $\begin{array}{c}0 \\
0 \\
2(13.33)\end{array}$ & $\begin{array}{l}0.05 \\
0.18 \\
0.49\end{array}$ \\
\hline \multicolumn{4}{|c|}{ Nonspecific features } \\
\hline Sinusoidal dilatation & $16(35.56)$ & $7(46.67)$ & 0.54 \\
\hline $\begin{array}{l}\text { Para-portal shunt vessels } \\
\text { Increased vascular channels in PT }\end{array}$ & $\begin{array}{c}9(20) \\
5(11.11)\end{array}$ & $\begin{array}{c}0 \\
2(13.33)\end{array}$ & $\begin{array}{c}0.095 \\
1\end{array}$ \\
\hline PV dilatation & $4(8.89)$ & $4(26.67)$ & 0.09 \\
\hline Increased parenchymal draining vessels & $1(2.22)$ & 0 & 1 \\
\hline Perivenular fibrosis & $1(2.22)$ & 0 & 1 \\
\hline
\end{tabular}

(PT: Portal tract, PV: Portal vein, PHT: Portal hypertension)

Table 2: Cases with septal fibrosis $(n=5)$ :

\begin{tabular}{|l|l|l|l|l|l|}
\hline C/F and histology & Case 1 & Case 2 & Case 3 & Case 4 & Case 5 \\
\hline Age/Sex & 22/F & 29/F & $23 / F$ & $49 / F$ & $46 / F$ \\
\hline Duration of symptoms & 1 year & 1 year & 4 years & 7 months & 3 months \\
\hline PTINR & 1.14 & 1.32 & 1.1 & 0.9 & 1.1 \\
\hline Fibrosis stage & $5 / 6(H S F)$ & $3 / 6(H S F)$ & $3 / 6(H S F)$ & $1 / 6(L S F)$ & $3 / 6(H S F)$ \\
\hline Follow up & 2 years & 11 years & 2 years & 3 years & 6 months \\
\hline
\end{tabular}

(C/F-Clinical features, HSF: High stage fibrosis, LSF: Low stage fibrosis) cases with high stage fibrosis, as well as those presenting with decompensation.

Of the 15 controls, (EHPVO cases), their age ranged from 9 years to 36 years (mean age 20) with slight female preponderance (M: F - 1:1.5). All presented with splenomegaly with esophageal varices in most cases. On histology (table 1), most common findings were phlebosclerosis and portal tract fibrosis. Other nonspecific features were sinusoidal and portal vein dilatation and increased portal vascular channels seen in few cases. Occasional case also showed recanalised thrombus in portal vein (Figure $2 \mathrm{~F}$ ). 

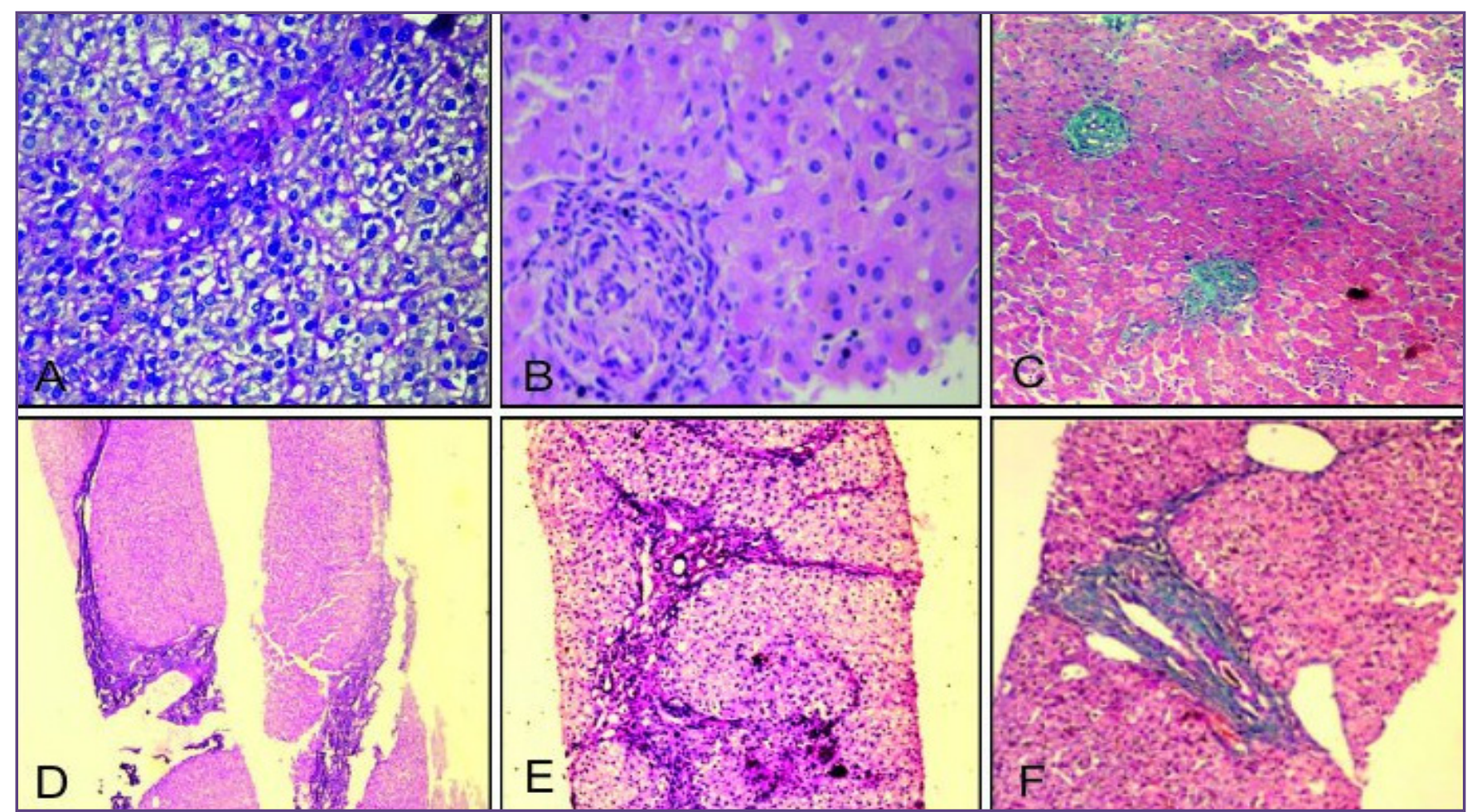

Fig. 1: Fibrotic portal tracts with obliterated small portal vein (A and B: H \&E X400 and C: MT x100). Higher stage septal fibrosis (D: H\&E x 40, E: H\&E x 100, and F: MT x 100)
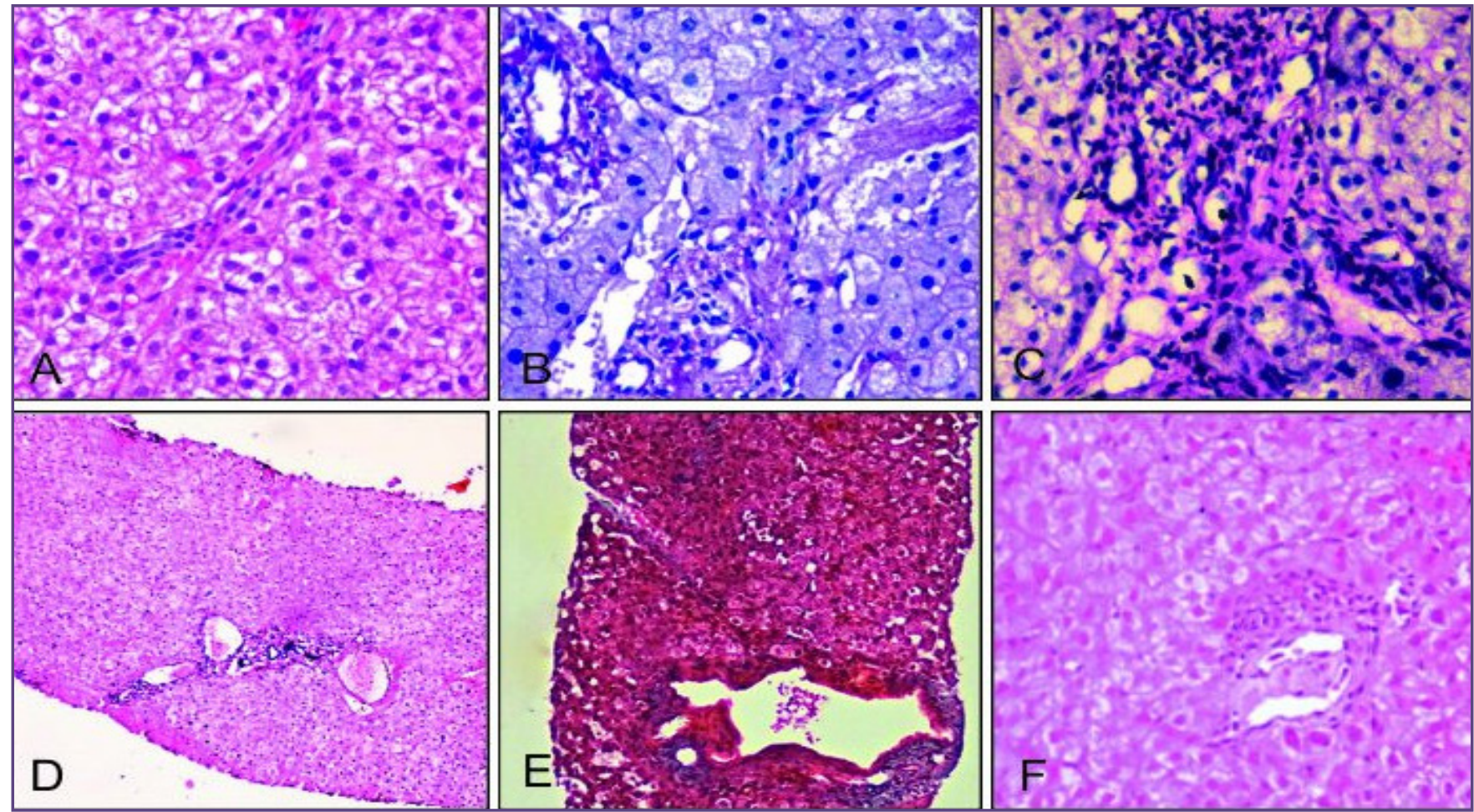

Fig. 2: Portal tract remnant, approximation and increased in number of thin-walled vessels (A, B, C: H\&E x400). Para-portal shunt vessels and Portal vein dilatation (D,E: H\&E, MT x100). Recanalised thrombus in portal vein in EHPVO (F: H \& E, x400) 


\section{Discussion}

Non-cirrhotic portal hypertension presents in a significant group of patients and accounts for $15-30 \%$ of all patients of portal hypertension. In our study of 45 cases of NCPF (average 4.5 cases per year), there was slight female preponderance with mean age being 34.3 years and duration varying from 3 months to 11 years. The study by Dhiman et al from north India $(\mathrm{n}=151)^{[10]}$ and Okuda et al from Japan $(\mathrm{n}=122){ }^{[21]}$ also noted slight female preponderance. However, other studies from India such as Pandey et al $(n=336)$ from South India ${ }^{[16]}$, Sarin et al $(n=326)$ from North India ${ }^{[18]}$ found equal M: $F$ ratio. This could be due to difference in sample size and/ or regional variation; however, age of presentation was similar in all these studies. In contrast, study by Verheji et al $(n=70)$ from west (Netherland and Belgium) ${ }^{[13]}$ reported male preponderance with mean age of presentation being 10 years higher.

In our study, splenomegaly, esophageal variceal bleeding and anemia were the common presenting symptoms. Similar symptoms were also reported in other studies too. $[10,13,17,21]$ Jaundice and ascites were seen in few in our study, similar to those reported by Dhiman et al and Madhu et al. ${ }^{[10,17]}$

The parenchymal damage is minimal in NCPF which is manifests as near normal/ minimal rise in aminotransferase levels. ${ }^{[3]}$ Various other studies from India, Japan as well as from western countries have also reported the same. $[10,13,16,17,21]$ In our study too, most patients showed normal aminotransferases, while only few showed minimal to moderate rise of levels (range 43-90 U/L).

Consensus definition according to Asian Pacific Association of Study of Liver is 'It is a disease of uncertain etiology characterized by periportal fibrosis with involvement of small and medium sized portal vein branches, resulting in the development of portal hypertension' ${ }^{\left[{ }^{[8]}\right.}$ The histological changes in NCPF were aptly summarized by Nayak et al. as 'obliterative porto-venopathy of liver'.$^{[11]}$ In our study, commonest histological features were portal tract fibrosis, phlebosclerosis and portal tract remnants similar to that described by others. It is possible that some authors might have described portal tract remnants as streaky portal tract/ pinched off portal tract, while others have interpreted phlebosclerosis as portal tract fibrosis..$^{[10,13,17]}$ In our study, normal histology was also seen in few cases, which is similar to the findings of Madhu et al, ${ }^{[17]}$ but not described by others.

The septal fibrosis in NCPF are not as common as seen in cirrhosis, but thin incomplete septae (low stage fibrosis) have been described in various studies. ${ }^{[17,24,25,26]}$ In our study too, septal fibrosis were seen in 5. However, septae were complete (high stage fibrosis, $\geq$ stage 3 ) in most $(n=4)$. Okuda et al $(n=122)^{[21]}$ and Madhu et al $(n=30)^{[17]}$ in their study have described septal fibrosis in approximately $52 \%$ and $6.66 \%$ cases respectively, but on wedge biopsies. Dhiman et al $(\mathrm{n}=100)$ have also reported septal fibrosis in $10 \%$ cases $(\mathrm{n}=10)$ of which only 7 were needle biopsies, however they did not describe the extent of fibrosis in detail in these needle biopsies. ${ }^{[10]}$ Thus most studies have reported the description of fibrosis on wedge biopsies, which is not ideal as it is known to show subcapsular scarring and nodularity, regularly in routine samples. ${ }^{[27,28]}$ Hence, the needle biopsies are recommended, as were done in our study. We also did staging of these cases as per Ishak's modification of hepatitis activity index (HAI) system for chronic hepatitis, ${ }^{[20]}$ which is not done by other authors to the best of our knowledge. However, it still remains the matter of discussion, whether the same system should be applied to stage NCPF/IPH/INCPH as well. Also all the patients with septal fibrosis in our study were female, thus we need to study further, whether females are more prone to develop fibrosis or it simply just followed the trend of overall female dominance as was present in our study (M: F - 1:1.5).

The course of NCPF is usually benign. It rarely presents with the features of decompensation and liver failure. ${ }^{[29,30,31,32]}$ Same is reported by Dhiman et al ${ }^{[10]}$ and Madhu et $\mathrm{al}^{[17]}$ too in $10 \%$ cases. In a French follow-up study, PVT, ascites and liver failure have been shown to develop in $46 \%, 50 \%$, and $21 \%$, respectively, over a mean period of 7.6 years. ${ }^{[33]}$ In our study also, decompensation was seen in few, but their course remained stable on further follow up, including those showing higher stage fibrosis. However, this kind of follow up was not available in other studies.

EHPVO and NCPF share etiological factors, clinical presentation and histological features. Early age acute or recurrent infections in an individual with thrombotic predisposition constitute the likely pathogenesis and both disorders present with clinically significant PHT with preserved liver functions. ${ }^{[33]}$ Most of the time, diagnosis of the former does not required liver biopsy, however there could be overlap of the features in some cases. ${ }^{[3,8,16]} \mathrm{We}$ analyzed and tried to compare clinical and histological features of EHPVO and NCPF cases (Table 1). In our study, EHPVO presented at younger age but showed M: F ratio similar to NCPF. Both presented with splenomegaly and esophageal varices. However on histology, among specific features, phlebosclerosis, portal tract fibrosis and portal tract remnants were significantly more frequent with NCPF of which of phlebosclerosis was found to be statistically significant $(\mathrm{p}=0.0001)$. 


\section{Conclusion}

Thus to summarize, NCPF still remains one of the important causes of portal hypertension in India in spite of improvement in hygiene and living standards. It can present with features of decompensation mimicking cirrhosis, but have relatively stable course. Phlebosclerosis, portal tract fibrosis and portal tract remnants still remain most common histological findings with phlebosclerosis being statistically significant when comparing with the features of EHPVO. A high stage fibrosis ( $\geq$ stage 3 ) can also be present in NCPF, however exclusive study of a larger series of only those cases showing septal fibrosis should be done along with long term follow up, to differentiate it from cryptogenic cirrhosis. A multicentric collaboration is necessary for any further conclusion.

\section{Acknowledgements}

Dr Shobhna Bhatia, Professor and Head, Department of Gastroenterology, Seth G.S. Medical College \& KEM Hospital, Mumbai.

\section{Reference}

1. Sanyal AJ, Bosch J, Blei A, Arroyo V. Portal hypertension and its complications. Gastroenterology 2008;134: 1715 -1728 .

2. Sarin SK, Kumar A. Noncirrhotic portal hypertension. Clin Liver Dis 2006;10: 627-651.

3. Sarin SK, Kumar A, Chawla YK, Baijal SS, Dhiman RK, Jafri $W$ et al. Non-cirrhotic portal fibrosis/idiopathic portal hypertension: APASL recommendations for diagnosis and treatment. Hepatol Int 2007;1: 398 - 413.

4. Sarin SK, Agarwal SR. Extrahepatic portal vein obstruction. Semin Liver Dis 2002; 22: 43 - 58.

5. Garcia-Pagan JC, Hernandez-Guerra M, Bosch J. Extrahepatic portal vein thrombosis. Semin Liver Dis 2008; 28: 282 - 292.

6. Yachha SK. Portal hypertension in children: an Indian perspective. J Gastroenterol Hepatol 2002; 17: S228-S231.

7. Poddar U, Thapa BR, Rao KL, Singh K. Etiological spectrum of esophageal varices due to portal hypertension in Indian children: is it different from the West? J Gastroenterol Hepatol 2008; 23:1354 - 1357.

8. Sarin SK, Sollano JD, Chawla YK, Amarapurkar D, Hamid S, Hashizume M. et al. Members of the APASL working party on portal hypertension. Consensus on extra-hepatic portal vein obstruction. Liver Int 2006; 26: 512 - 519.

9. Schouten JNL, Garcia-Pagan JC, Valla DC, Janssen HLA. Idiopathic noncirrhotic portal hypertension.Hepatology 2011; 54:1071 - 1081 .

10. Dhiman RK, Chawla Y, Vasishta RK et al. Non-cirrhotic portal fibrosis (idiopathic portal hypertension): experience with 151 patients and a review of the literature. J Gastroenterol Hepatol 2002;17: 6 - 16.
11. Nayak NC, Ramalingaswami B. Obliterative portal venopathy of the liver. Arch Pathol Med 1969; 87: 359 - 369.

12. Aoki H, Hasumi A, Yoshida K. A questionnaire study on treatment of idiopathic portal hypertension and extrahepatic portal obstruction. In: Kameda H, editor. Annual report on portal portal hemodynamics abnormalities (in Japanese). Tokyo: Japan Ministry of Health and Welfare; 1988: 179 189.

13. Verheij J, Schouten JNL, Komuta M, Nevens F, Hansen $\mathrm{BE}$, et al. Histological features in western patients with idiopathic non-cirrhotic portal hypertension. J Histopathol. 2013; 62:1083 -1091.

14. Vakili C, Farahvash MJ, Bynum TE. Endemic idiopathic portal hypertension. Report on 32 patients with non-cirrhotic portal fibrosis. World J Surg 1992;16:118 - 125.

15. Sarin SK, Agarwal SR. Idiopathic portal hypertension. Digestion 1998; 59: 420 - 423.

16. Pande C, Kumar A, Sarin SK. Non-cirrhotic portal fibrosis: a clinical profile of 366 patients. Am J Gastroenterol 2006;101:191.

17. Madhu K, Avinash B, Ramakrishna B, Eapen CE, Shyamkumar NK et al. Idiopathic non-cirrhotic intrahepatic portal hypertension: common cause of cryptogenic intrahepatic portal hypertension in a Southern Indian tertiary hospital. Indian J Gastroenterol 2009; 28: 83 - 87.

18. Sarin SK. Non-cirrhotic portal fibrosis. J Gastroenterol Hepatology. 2002; 17: S214 - S223.

19. Bernard $\mathrm{PH}$, Le Bail B, Cransac $\mathrm{M}$ et al. Progression from idiopathic portal hypertension to incomplete septal cirrhosis with liver failure requiring liver transplantation. J. Hepatol. 1995; 22: 495 - 499.

20. Ishak K, Baptista A, Bianchi L, Callea F, De Groote J, Gudat F, Denk H, Desmet V, Korb G, MacSween RN, et a. Histological grading and staging of chronic hepatitis. J Hepatol. 1995 Jun; 22: 696 - 699.

21. Kunio Okuda, Toshiro Nakashima, Masahiko Okudaira, Masayoshi Kage, Yoshifumi Aida, Masao Omata et al. Liver pathology of idiopathic portal hypertension. Comparison with non-cirrhotic portal fibrosis of India. Liver 1982; 2: $176-192$.

22. Nakanuma Y, Tsuneyama K, Ohbu M, Katayanagi K. Pathology and pathogenesis of idiopathic portal hypertension with an emphasis on the liver. Pathol Res Pract 2001; 197: $65-76$.

23. Okudaria $\mathrm{M}$, Ohbu $\mathrm{M}$, Okuda K. Idiopathic portal hypertension and its pathology. Semin Liver Dis 2002; 22: $59-71$.

24. Baldus WP. Idiopathic portal hypertension: a histopathologic study of 26 Japanese cases. Histopathology 1993; 22: 22734.

25. Sciot R, Staessen D, Van Damme B et al. Incomplete septal cirrhosis: histopathological aspects. Histopathology 1988; 13: 593 - 603 . 
26. Nevens F, Staessen D, Sciot R et al. Clinical aspects of incomplete septal cirrhosis in comparison with macronodular cirrhosis. Gastroenterology 1994; 106: 459 - 63.

27. James M. Crawford. Cirrhosis. In: Robert D. Odze and John R. Goldblum editors. Surgical Pathology of GI Tract, Liver, Biliary Tract and Pancreas, 3rd edition. SAUNDERS, an imprint of Elsevier Inc. Lmt.; 1978 - 79.

28. Petrelli M, Scheuer PJ. Variation in subcapsular liver structure and its significance in the interpretation of wedge biopsies. J Clin Pathol, 1967; 20: 743 - 748.

29. Hillaire S, Bonte E, Denninger $\mathrm{MH}$ et al. Idiopathic noncirrhostic intrahepatic portal hypertension in the west: a reevaluation in 28 patients. Gut 2002; 51: $275-80$.
30. Madhu K, Ramakrishna B, Zachariah U, Eapen CE, Kurian G. Non-cirrhotic intrahepatic portal hypertension. Gut 2008; $57: 152-9$.

31. Krasinskas AM, Eghtesad B, Kamath PS, Demetris AJ, Abraham SC. Liver transplantation for severe intrahepatic non-cirrhotic portal hypertension. Liver Transpl 2005; 11: 627- 34 .

32. Sarin SK, Kappor D. Non-cirrhotic portal fibrosis: current concepts and management. J Gastroenterol Hepatol 2002; 17: $526-34$.

33. Khanna R, Sarin SK. Non-cirrhotic portal hypertension Diagnosis and management. Journal of Hepatology 2014; 60:421-441

*Corresponding author:

Dr. Rachana Chaturvedi, 1203 Erica, Dosti Acres, Wadala East, Mumbai (India), ZIP CODE: 400037

Phone: +91 9967017267

Email: rachanachaturvedi@yahoo.co.in

Financial or other Competing Interests: None. 